\title{
Research Article Synchronization of the Extended Bonhoffer-Van der Pol Oscillators
}

\author{
Mohamed Zribi and Saleh Alshamali \\ Department of Electrical Engineering, Kuwait University, P.O. Box 5969, Safat 13060, Kuwait \\ Correspondence should be addressed to Mohamed Zribi, mohamed.zribi@ku.edu.kw
}

Received 19 February 2012; Revised 4 May 2012; Accepted 8 May 2012

Academic Editor: Jun-Juh Yan

Copyright (C) 2012 M. Zribi and S. Alshamali. This is an open access article distributed under the Creative Commons Attribution License, which permits unrestricted use, distribution, and reproduction in any medium, provided the original work is properly cited.

This paper deals with the synchronization of two extended Bonhoffer-Van der Pol (B-VDP) oscillators. A Lyapunov-based controller and a sliding mode controller are proposed for the synchronization of the oscillators. Both design schemes use a single input controller acting on one state only. Asymptotic stability results for the closed-loop system are derived using Lyapunov theory. It is shown that the proposed controllers effectively synchronize the driver and the response systems for the case when nominal values of the system parameters are used, and for the case when parameter perturbations are introduced. Simulation results are presented to show the effectiveness of the proposed controllers.

\section{Introduction}

Chaotic systems represent an interesting class of nonlinear systems. They are known to be highly sensitive to small changes in the initial conditions [1]. In the past two decades, the problem of synchronization and antisynchronization of chaotic systems has attracted a lot of interest. The synchronization phenomenon has been used in several key applications such as network security [2] and biological oscillators [3].

Several control strategies were suggested for the synchronization and anti-synchronization of chaotic systems; the suggested strategies include backstepping control, adaptive control, and active control. The results of some of these works are discussed below. In [4], a Lyapunov-based controller is developed to control the chaotic Duffing equation; the controller incorporates an observer that helps overcome parametric as well as external disturbances. An adaptive backstepping control law which is designed to control a class of chaotic systems is given in [5]; the chaotic system is transformed into a nonautonomous strict feedback form, then the developed backstepping controller is applied to asymptotically track a given reference signal. In [6], an integral sliding mode controller (SMC) with a dynamic 
sliding surface is proposed for the control of an extended Duffing-Van der Pol oscillator. The work in [7] proposes a combination of a state-feedback control and an adaptive control to synchronize a Van der Pol oscillator coupled with a linear oscillator. A state-feedback controller is used to stabilize the equilibrium point, then the adaptive control is utilized to synchronize two of such oscillators. In [8], a variable structure controller (VSC) is utilized to synchronize two chaotic systems. The chaotic systems are first transformed into the canonical form using the theory of differential geometry, then chaos suppression is achieved by applying the proposed controller. The performances of an active controller and a backstepping controller in synchronizing a class of chaotic systems are compared in [9]. It is shown that both control schemes have an acceptable transient performance. However, the active controller is more complex than the backstepping controller. In [10], a sliding mode controller is used in the control and synchronization of a class of uncertain chaotic systems, where the controller incorporates an adaptation law to ensure perfect synchronization. A nonlinear feedback controller, proposed for the synchronization of a class of chaotic systems known as the Lienard chaotic system, is given in [11]. The control design is based on Lyapunov theory. Sufficient conditions ensuring asymptotic stability are given in terms of the gains of the controller. In [12], an active control scheme is used for the synchronization and antisynchronization of the extended Bonhoffer-Van der Pol oscillators. The synchronization of a class of uncertain chaotic systems via sliding mode control is presented in [13]; the controller utilizes a disturbance observer based on a Radial Basis Function (RBF) neural network. The work in [14] proposes a nonlinear controller for the synchronization of a Van der Pol oscillator with a Chen chaotic circuit.

In this work, the extended Bonhoffer-Van der Pol (B-VDP) oscillator model presented in [12] is considered. A Lyapunov-based controller and a sliding mode controller (SMC) are proposed for the synchronization of the two chaotic B-VDP oscillators. Sliding mode control is well known for its fast response and disturbance rejection properties $[15,16]$. Unlike the work in [12] where three control actuators are used, in this work, a single control law is fed to the response system and designed to achieve complete synchronization between the drive and the response systems. Furthermore, for the proposed SMC design, a transformation is introduced to transform the error dynamics into a canonical form. The transformation greatly simplifies the design of the controller.

The paper is organized as follows. The model of the extended B-VDP oscillator circuit is introduced in Section 2. Section 3 presents a Lyapunov-based controller to synchronize two B-VDP oscillators. The design of a sliding mode controller to synchronize the two B-VDP Oscillators is given in Section 4. Simulation results are given and discussed in Section 5. Finally, some concluding remarks are given in Section 6.

\section{The Model of the Extended Bonhoffer-Van der Pol Oscillator}

The extended B-VDP oscillator circuit consists of two capacitors, an inductor, a linear resistor and a nonlinear resistor (see Figure 1). Its dynamic model can be described by the following set of ordinary differential equations [12]:

$$
C \frac{d v_{1}}{d t}=-i-g\left(v_{1}\right)
$$




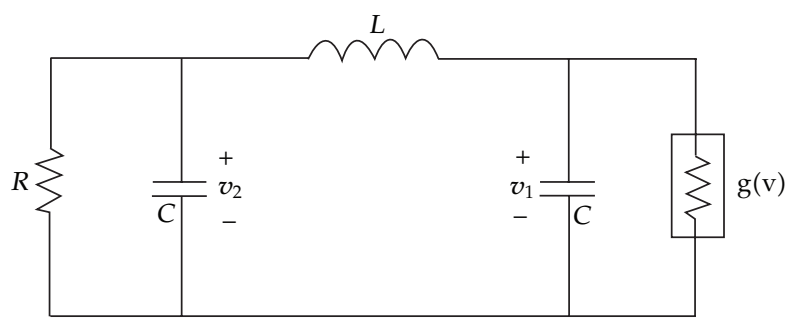

Figure 1: The extended B-VDP oscillator circuit.

$$
\begin{aligned}
& C \frac{d v_{2}}{d t}=i-\frac{v_{2}}{R}, \\
& L \frac{d i}{d t}=v_{1}-v_{2} .
\end{aligned}
$$

The states of system (2.1) are $v_{1}, v_{2}$, and $i$; these states are defined as follows:

$v_{1}$ : the voltage across the first capacitor,

$v_{2}$ : the voltage across the second capacitor,

$i$ : the current through the inductor,

$R, C, L$ : the resistance, the capacitances, and the inductance in the circuit, $g\left(v_{1}\right)$ : the nonlinear resistor defined such that

$$
g\left(v_{1}\right)=-a v_{1}-b \tanh \left(c v_{1}\right)
$$

where $a$ and $b$ are parameters of the system. In order to facilitate the control design, the extended B-VDP dynamic model (2.1) is expressed in a normalized form by redefining the parameters and state variables of the system [12]. To this end, let the new time variable be given as $\tau=(1 / \sqrt{L C}) t$. Also, define the new state vector, $[x, y, z]^{T}$, such that $x=\left(v_{1} / b\right) \sqrt{C / L}, \quad y=\left(v_{2} / b\right) \sqrt{C / L}$, and $z=i / b$. Finally, define the parameters $p_{1}, p_{2}$, and $p_{3}$ such that $p_{1}=a \sqrt{L / C}, p_{2}=b c \sqrt{L / C}$, and $p_{3}=$ $(1 / R) \sqrt{L / C}$. Hence, the normalized model of the extended B-VDP oscillator is such that

$$
\begin{gathered}
\dot{x}=-z+p_{1} x+\tanh \left(p_{2} x\right), \\
\dot{y}=z-p_{3} y, \\
\dot{z}=x-y,
\end{gathered}
$$

where the "." denotes the differentiation with respect to $\tau$. 
Generally, synchronization involves two systems: the first system is known as the drive (master) system, and the second one is known as the response (slave) system. The dynamic model of the drive circuit of the B-VDP oscillator be given as

$$
\begin{gathered}
\dot{x}_{d}=-z_{d}+p_{1} x_{d}+\tanh \left(p_{2} x_{d}\right), \\
\dot{y}_{d}=z_{d}-p_{3} y_{d}, \\
\dot{z}_{d}=x_{d}-y_{d},
\end{gathered}
$$

where $x_{d}, y_{d}$, and $z_{d}$ are the states of the drive system. Moreover, the dynamic model of the response system can be written as follows:

$$
\begin{gathered}
\dot{x}_{r}=-z_{r}+p_{1} x_{r}+\tanh \left(p_{2} x_{r}\right)+u, \\
\dot{y}_{r}=z_{r}-p_{3} y_{r}, \\
\dot{z}_{r}=x_{r}-y_{r} .
\end{gathered}
$$

Note that the first differential equation of the response system contains the forcing term $u$. This term represents the controller of the system, which will be designed such that the drive system and the response system are synchronized after starting from different initial conditions. It is noted that the synchronization of the two systems is achieved through the use of a single input controller acting on one state only. Minimizing the number of actuators is good from an implementation point of view, as the implementation becomes cost effective.

For the control design, the error vector $e(t)=\left[\begin{array}{lll}e_{1}(t) & e_{2}(t) & e_{3}(t)\end{array}\right]^{T}$ is defined such that the errors between the states of the response system and the drive system are given by

$$
\begin{aligned}
& e_{1}=x_{r}-x_{d}, \\
& e_{2}=y_{r}-y_{d}, \\
& e_{3}=z_{r}-z_{d} .
\end{aligned}
$$

Using (2.4)-(2.6), the error dynamics can be written as

$$
\begin{gathered}
\dot{e}_{1}=-e_{3}+p_{1} e_{1}+\tanh \left(p_{2} x_{r}\right)-\tanh \left(p_{2} x_{d}\right)+u, \\
\dot{e}_{2}=e_{3}-p_{3} e_{2}, \\
\dot{e}_{3}=e_{1}-e_{2} .
\end{gathered}
$$

Obviously, complete synchronization between the drive system and the response system is achieved when the errors converge to zero. Therefore, the objective of this work is to design controllers which force the errors to asymptotically converge to zero as $t$ tends to infinity. In Sections 3 and 4, two control design methodologies are introduced to accomplish the synchronization task. 


\section{Design of a Lyapunov-Based Controller}

In this section, a Lyapunov-based controller is used to force the errors between the drive and the response circuits to zero as $t$ tends to infinity and hence synchronize the extended B-VDP oscillators.

Let $\alpha$ be a positive scalar.

Proposition 3.1. The control law:

$$
u=-\left(p_{1}+\alpha\right) e_{1}-\tanh \left(p_{2} x_{r}\right)+\tanh \left(p_{2} x_{d}\right)
$$

renders the error system (2.7) globally asymptotically stable.

Proof. Let the Lyapunov function candidate $V_{1}$ be such that

$$
V_{1}=\frac{1}{2} e_{1}^{2}+\frac{1}{2} e_{2}^{2}+\frac{1}{2} e_{3}^{2}
$$

Taking the time derivative of $V_{1}$, and using expressions (2.7) and (3.1) of the dynamic model of the errors and the control law, respectively, gives

$$
\begin{aligned}
\dot{V}_{1} & =e_{1} \dot{e}_{1}+e_{2} \dot{e}_{2}+e_{3} \dot{e}_{3} \\
& =e_{1}\left(-e_{3}+p_{1} e_{1}+\tanh \left(p_{2} x_{r}\right)-\tanh \left(p_{2} x_{d}\right)+u\right)+e_{2}\left(e_{3}-p_{3} e_{2}\right)+e_{3}\left(e_{1}-e_{2}\right) \\
& =-\alpha e_{1}^{2}-p_{3} e_{2}^{2} .
\end{aligned}
$$

Clearly, $V_{1}$ is a positive definite function defined over $\mathbb{R}^{3}$ such that $\dot{V}_{1}$ is negative semidefinite in $\mathbb{R}^{3}$. In order to show the asymptotic stability of the closed-loop system, we will use LaSalle's theorem [17]. Define the compact set $\Omega$ such that

$$
\Omega=\left\{e(t) \in \mathbb{R}^{3} \mid \dot{V}_{1}=0\right\}
$$

Solving the equation $\dot{V}_{1}=0$, and using the fact that $\alpha$ and $p_{3}$ are positive constants, we get

$$
\dot{V}_{1}=0 \Longrightarrow-\alpha e_{1}^{2}-p_{3} e_{2}^{2}=0 \Longrightarrow e_{1}(t)=0, \quad e_{2}(t)=0
$$

Note that $e_{1}(t)=0$ implies that $\dot{e}_{1}(t)=0$, and that $e_{2}(t)=0$ implies that $\dot{e}_{2}(t)=0$.

Now, using the second differential equation given in (2.7), it follows that

$$
\dot{e}_{2}(t)=0 \Longrightarrow e_{3}-p_{3} e_{2}=0 \Longrightarrow e_{3}(t)=0
$$

Therefore, the only solution of the equation $\dot{V}_{1}=0$ is $e_{1}(t)=0, e_{2}(t)=0$, and $e_{3}(t)=0$. This means that no solution can stay identically in $\Omega$ other than the trivial solution $(0,0,0)^{T}$. 
Hence, from LaSalle's invariance theorem, it can be concluded that the origin of the error system (2.7) is asymptotically stable. Finally, the Lyapunov function $V_{1}$ satisfies

$$
\lim _{\|e\| \rightarrow \infty} V_{1}=\infty
$$

Thus $V_{1}$ is radially unbounded and hence the origin is globally asymptotically stable. Therefore, it can be concluded that the control law (3.1) when applied to the error system (2.7) guarantees the convergence of the errors $e_{1}, e_{2}$, and $e_{3}$ to zero as $t$ tends to infinity.

It should be noted that since the errors $e_{1}, e_{2}$, and $e_{3}$ asymptotically converge to zero as $t$ tends to infinity, then $x_{r}, y_{r}$, and $z_{r}$ converge to $x_{d}, y_{d}$, and $z_{d}$, respectively, as $t$ tends to infinity. Therefore, the states of the drive and the response chaotic systems are synchronized.

Remark 3.2. The parameter $\alpha$ in the control law (3.1) can be taken to be zero. However, the inclusion of the term $-\alpha e_{1}$ speeds up the convergence of the error trajectories to zero.

\section{Design of a Sliding Mode Controller}

This section deals with the design of a sliding mode controller to synchronize two extended B-VDP oscillators. To facilitate the design of the controller, the following transformation is introduced:

$$
\begin{gathered}
\zeta_{1}=e_{2} \\
\zeta_{2}=e_{3}-p_{3} e_{2} \\
\zeta_{3}=e_{1}+\left(p_{3}^{2}-1\right) e_{2}-p_{3} e_{3} .
\end{gathered}
$$

The inverse of this transformation is defined for all values of $p_{3}$ and it is such that:

$$
\begin{gathered}
e_{1}=\zeta_{1}+p_{3} \zeta_{2}+\zeta_{3}, \\
e_{2}=\zeta_{1}, \\
e_{3}=p_{3} \zeta_{1}+\zeta_{2} .
\end{gathered}
$$

Using the transformation (4.1), the error dynamics given by (2.7) can be written using the new coordinates as

$$
\begin{gathered}
\dot{\zeta}_{1}=\zeta_{2}, \\
\dot{\zeta}_{2}=\zeta_{3}, \\
\dot{\zeta}_{3}=f+u,
\end{gathered}
$$


where the function $f$ is given by

$$
f=-e_{3}+p_{1} e_{1}+\left(p_{3}^{2}-1\right)\left(e_{3}-p_{3} e_{2}\right)-p_{3}\left(e_{1}-e_{2}\right)+\tanh \left(p_{2} x_{r}\right)-\tanh \left(p_{2} x_{d}\right) .
$$

Remark 4.1. The function $f$ can be written using the $\zeta_{1}, \zeta_{2}, \zeta_{3}$ coordinates such that:

$$
f=\left(p_{1}-p_{3}\right) \zeta_{1}+\left(p_{1} p_{3}-2\right) \zeta_{2}+\left(p_{1}-p_{3}\right) \zeta_{3}+\tanh \left(p_{2} x_{r}\right)-\tanh \left(p_{2} x_{d}\right)
$$

The first step in the design of a sliding mode controller is to define a sliding surface towards which the system trajectories must be forced. We will define the linear sliding surface $\sigma$ such that:

$$
\sigma=\zeta_{3}+\beta_{1} \zeta_{1}+\beta_{2} \zeta_{2}
$$

where $\beta_{1}$ and $\beta_{2}$ are positive scalars such that the polynomial $P(x)=s^{2}+\beta_{2} s+\beta_{1}$ is Hurwitz. The derivative of $\sigma$ with respect to time is given by

$$
\begin{aligned}
\dot{\sigma} & =\dot{\zeta}_{3}+\beta_{1} \dot{\zeta}_{1}+\beta_{2} \dot{\zeta}_{2} \\
& =f+\beta_{1} \zeta_{2}+\beta_{2} \zeta_{3}+u
\end{aligned}
$$

The objective is to design a control law, $u$, such that the system trajectories reach the sliding surface in finite time and stay there for all subsequent time. Define the sign function such that

$$
\operatorname{sign}(\sigma)= \begin{cases}1 & \text { if } \sigma>0 \\ 0 & \text { if } \sigma=0 \\ -1 & \text { if } \sigma<0\end{cases}
$$

Also, let $K$ and $W$ be positive scalars.

Proposition 4.2. The sliding mode controller:

$$
u=-f-\beta_{1} \zeta_{2}-\beta_{2} \zeta_{3}-K \sigma-W \operatorname{sign}(\sigma)
$$

renders the transformed error system (4.3) asymptotically stable.

Proof. First, we will show that the proposed controller forces the dynamics of the system to reach the sliding surface in finite time. Then, the closed-loop stability of the system on the sliding surface is proved. To prove the reachability to $\sigma=0$, it suffices to show that $\sigma \quad \dot{\sigma}<0$. To this end, consider the Lyapunov function candidate $V_{2}=(1 / 2) \sigma^{2}$. Taking the derivative of 


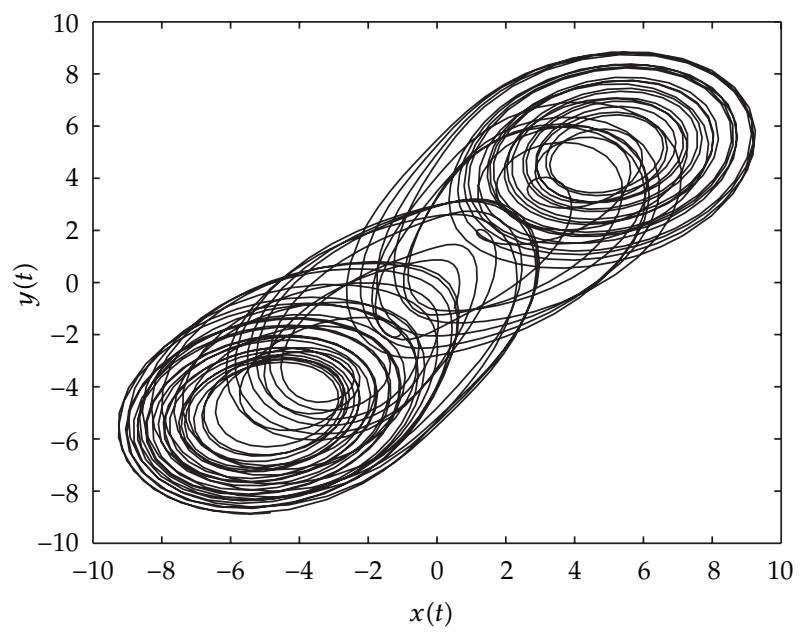

Figure 2: Plot of $x(t)$ versus $y(t)$ with no control.

$V_{2}$ with respect to time and using the expressions (4.7) and (4.9) for $\dot{\sigma}$ and $u$, respectively, it follows that

$$
\begin{aligned}
\dot{V}_{2} & =\sigma\left(f+\beta_{1} \zeta_{2}+\beta_{2} \zeta_{3}+u\right) \\
& =\sigma(-K \sigma-W \operatorname{sign}(\sigma)) \\
& =-K \sigma^{2}-W|\sigma| .
\end{aligned}
$$

Since $K$ and $W$ are chosen to be positive scalars, it follows that $\dot{V}_{2}<0$ for $\sigma \neq 0$ and $\dot{V}_{2}=0$ for $\sigma=0$. Hence $V_{2}$ is a Lyapunov function for the system. Therefore, it can be concluded that the finite time reachability to the surface $\sigma=0$ is guaranteed. Note that upon reaching the sliding surface, we have $\sigma=0$ and $\dot{\sigma}=0$.

On the sliding surface $(\sigma=0)$, the state $\zeta_{3}$ can be written as $\zeta_{3}=-\beta_{1} \zeta_{1}-\beta_{2} \zeta_{2}$. Therefore, the closed-loop dynamics of the reduced order system (i.e., the system when $\sigma=0$ ) can be written as

$$
\begin{gathered}
\dot{\zeta}_{1}=\zeta_{2} \\
\dot{\zeta}_{2}=-\beta_{1} \zeta_{1}-\beta_{2} \zeta_{2}
\end{gathered}
$$

Since $\beta_{1}$ and $\beta_{2}$ are chosen to be positive scalars such that the polynomial $P(x)=s^{2}+\beta_{2} s+\beta_{1}$ is Hurwitz, it can be concluded that the states $\zeta_{1}$ and $\zeta_{2}$ will converge to zero asymptotically as $t$ tends to infinity. Moreover, since $\zeta_{3}=-\beta_{1} \zeta_{1}-\beta_{2} \zeta_{2}$, it follows that $\zeta_{3}$ will asymptotically converge to zero as $t$ tends to infinity.

It is clear from the transformation (4.1) that the asymptotic convergence of $\zeta_{1}, \zeta_{2}$ and $\zeta_{3}$ to zero implies the asymptotic convergence of the errors $e_{1}, e_{2}$, and $e_{3}$ to zero. Using (2.6), it is noted that when the errors $e_{1}, e_{2}$ and $e_{3}$ converge to zero as $t$ tends to infinity, then the convergence of $x_{r}, y_{r}$, and $z_{r}$ to $x_{d}, y_{d}$, and $z_{d}$, respectively, is guaranteed as $t$ tends to infinity. Therefore, the states of the drive and the response chaotic systems are synchronized. 


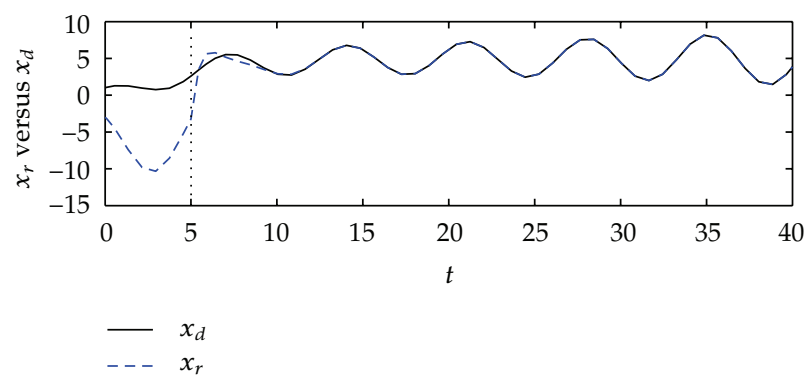

(a)

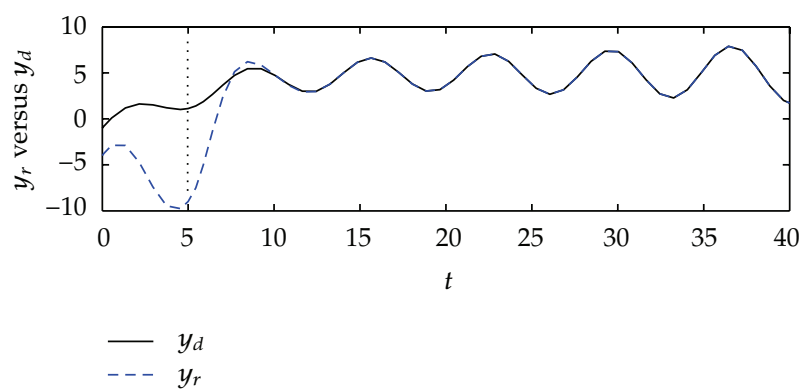

(b)

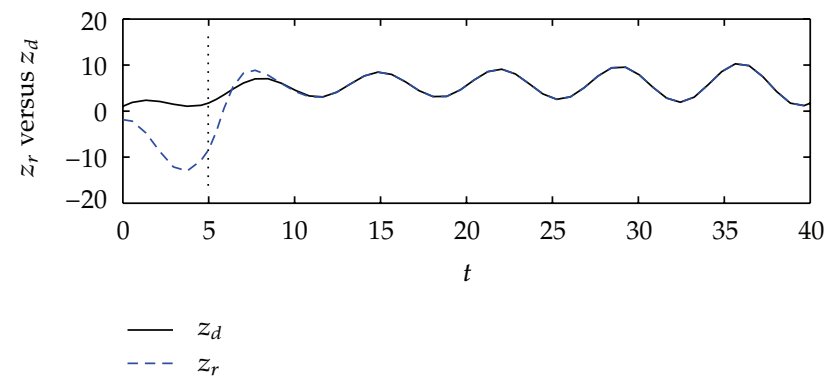

(c)

Figure 3: Plots of the extended B-VDP oscillators' state trajectories versus time when the Lyapunov-based controller is used.

Remark 4.3. To reduce or eliminate the chattering associated with the proposed sliding mode controller, we can use the hyperbolic tangent instead of the sign function. Moreover, chattering can be reduced or eliminated by using higher order sliding mode controllers which can be easily designed since the error system given by (4.3) is in the controller canonical form.

Remark 4.4. The term $-K \sigma(K>0)$ in (4.9) is added to speed up the convergence of the error trajectories.

\section{Simulation Results}

Several simulation studies are conducted to illustrate the effectiveness of the proposed controllers in synchronizing two extended B-VDP oscillators. First, a simulation of the extended B-VDP system under no control is carried out. Figure 2 shows the chaotic attractor of an 


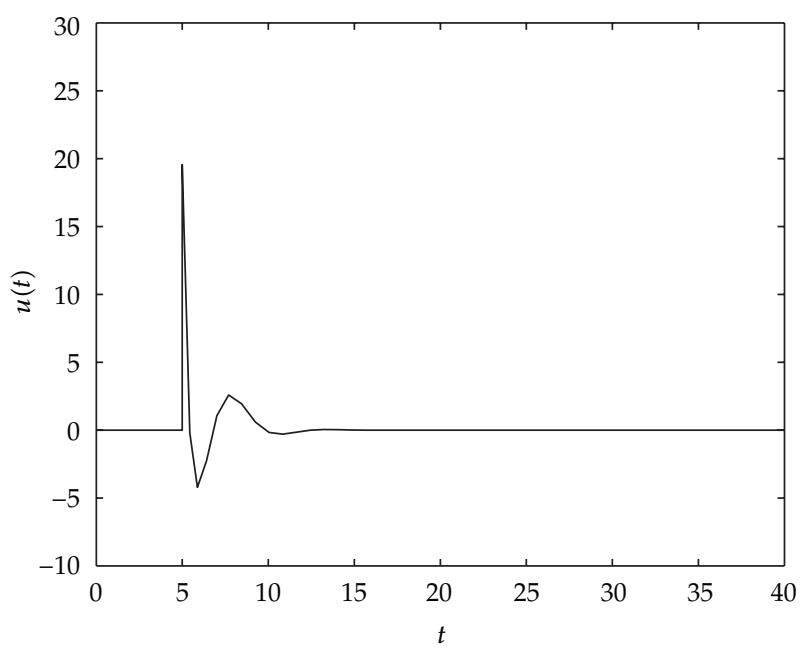

Figure 4: Plot of the Lyapunov-based controller versus time.

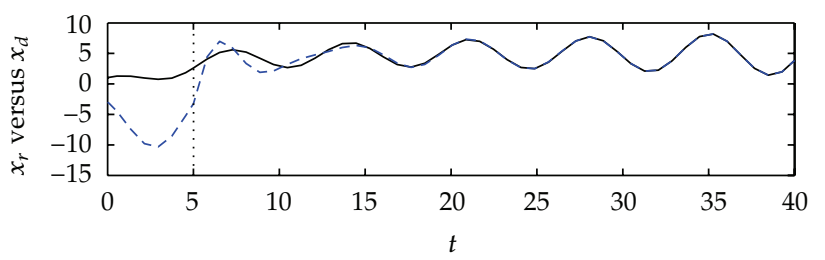

$$
\begin{array}{ll}
- & x_{d} \\
--- & x_{r}
\end{array}
$$

(a)

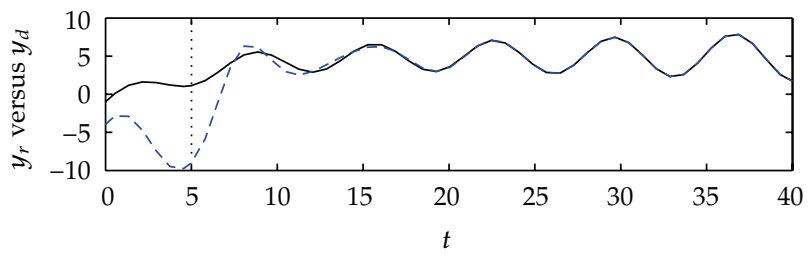

$$
-\begin{aligned}
& y_{d} \\
& --- \\
& y_{r}
\end{aligned}
$$

(b)

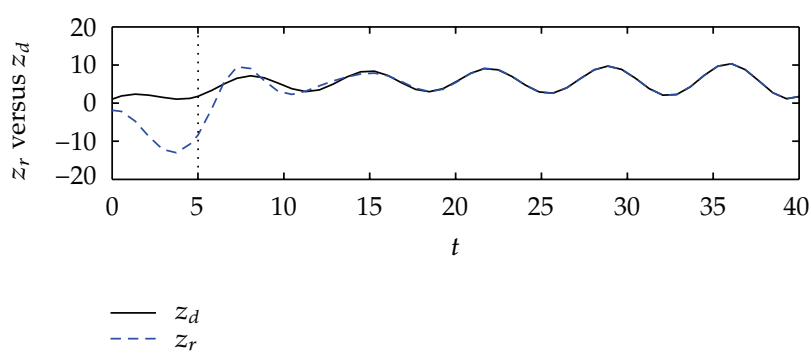

(c)

Figure 5: Plots of the extended B-VDP oscillators' state trajectories versus time when the Lyapunov-based controller is used and with $\alpha=0$. 


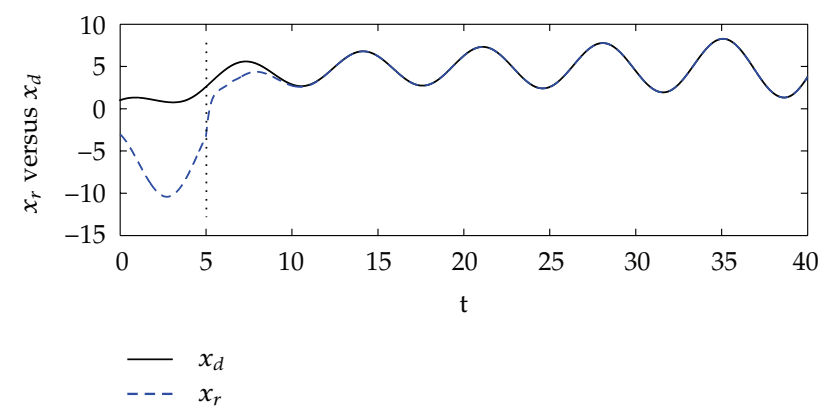

(a)

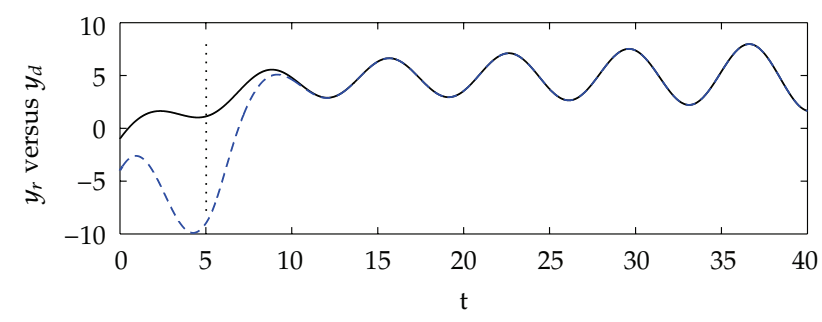

$-y_{d}$

(b)

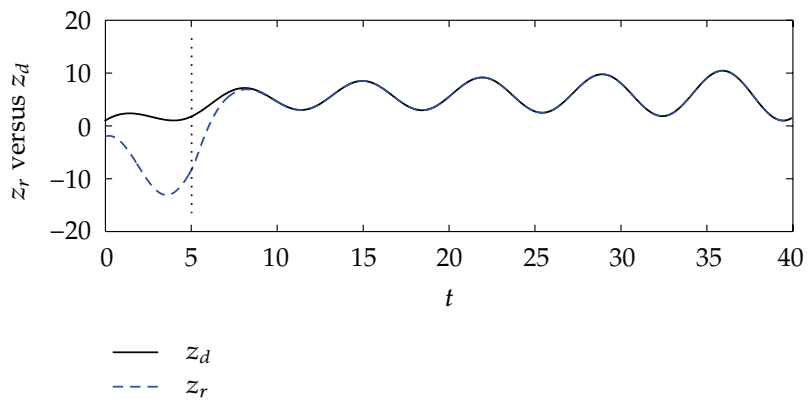

(c)

Figure 6: Plots of the extended B-VDP oscillators' state trajectories versus time when the sliding mode controller is used.

extended B-VDP oscillator for the case when the parameters of the system are $p_{1}=1, p_{2}=1$, and $p_{3}=1.2$, and for initial conditions $\left(x_{d}(0), y_{d}(0), z_{d}(0)\right)^{T}=(0.5,-1,0.5)^{T}$.

The performance of the Lyapunov-based controller used to synchronize the two extended B-VDP oscillators, given by (2.4) and (2.5), is shown in Figures 3 and 4 . The parameters of the oscillators are given by $p_{1}=1, p_{2}=1$, and $p_{3}=1.2$. The initial conditions of the drive system are taken to be $x_{d}(0)=1, y_{d}(0)=-1$ and $z_{d}(0)=1$; the initial conditions of the response system are taken to be $x_{r}(0)=-3, y_{r}(0)=-4$ and $z_{r}(0)=-2$. The gain of the controller $\alpha$ is chosen such that $\alpha=2$. Furthermore, the controller is applied to the response system at $t=5 \mathrm{sec}$. Figure 3 shows the trajectories of the drive and response systems. It is clear that complete synchronization takes place at about $t=9 \mathrm{sec}$. The vertical dotted lines in the figure indicate the time at which the controller is applied. Figure 4 shows the control action. 


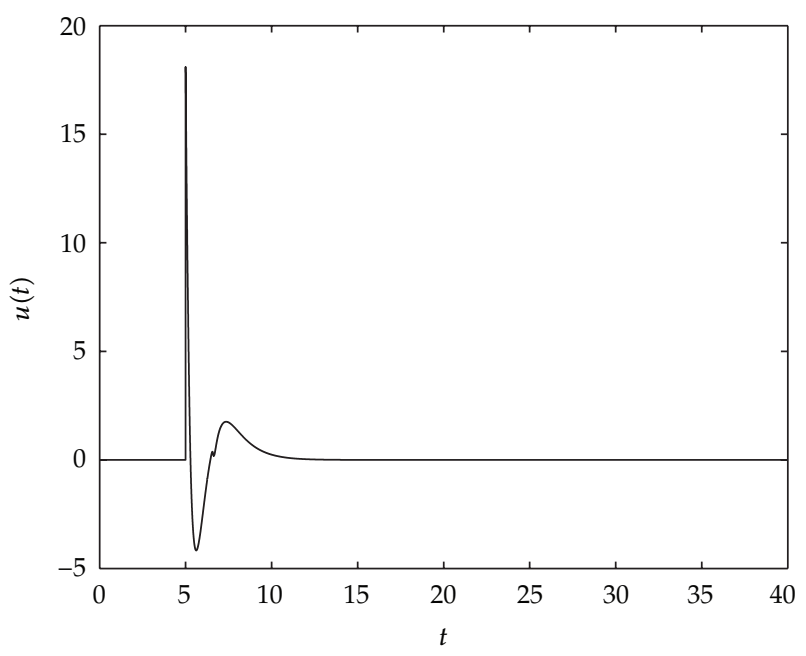

Figure 7: Plot of the sliding mode controller versus time.

A small spike appears when the controller is switched on. Finally, Figure 5 depicts the trajectories of the two oscillators for the case when the parameter $\alpha$ is equal to zero. As indicated in Remark 3.2, this parameter can be taken to zero at the expense of the speed of the convergence of the errors to zero. Figure 5 indicates that the synchronization is attained at around $t=20$ seconds (or 15 seconds after the controller is switched on).

The performance of the sliding mode controller used to synchronize the extended BVDP oscillators is depicted in Figures 6 and 7. The initial conditions are the same as the ones used previously. The parameters of the controller are chosen such that $K=2, W=1, \beta_{1}=2$, and $\beta_{2}=3$. Figure 6 depicts the evolution of the state trajectories of the drive and response systems. Synchronization is achieved 4 seconds after the controller is applied. Figure 7 depicts the control action trajectory, where a spike appears at the time when the controller is applied at $t=5 \mathrm{sec}$.

Finally, in order to check the robustness of the proposed sliding mode control scheme, a $5 \%$ change on the parameters $p_{1}, p_{2}$, and $p_{3}$ is introduced. Figure 8 depicts the results of this case. The two oscillators synchronize few seconds after the controller is applied.

Therefore, it can be concluded from the simulation results that the proposed controllers work well as they are able to synchronize the drive and the response systems. Also, the robustness of the sliding mode controller under parameter perturbations is evident from the simulation results.

\section{Conclusion}

A Lyapunov-based controller and a sliding mode controller are proposed for the synchronization of two extended Bonhoffer-Van der Pol oscillators which start from different initial conditions. The single input controller acts on the first state of the response system only, which makes it easier to implement. It is shown that the proposed controllers guarantee the asymptotic convergence to zero of the errors between the response and the drive systems, hence, 


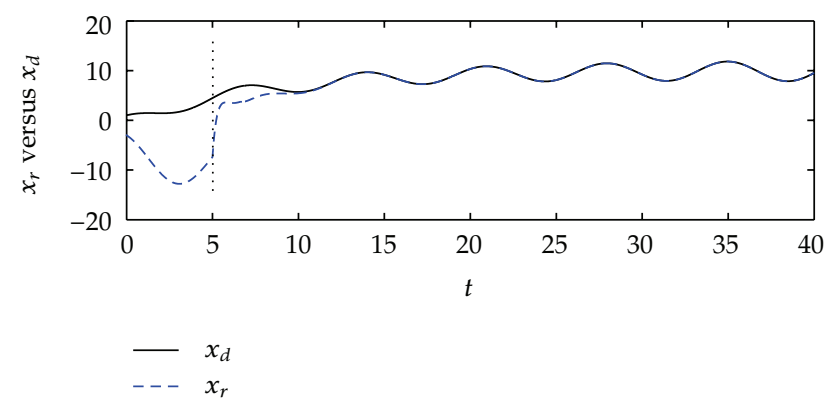

(a)

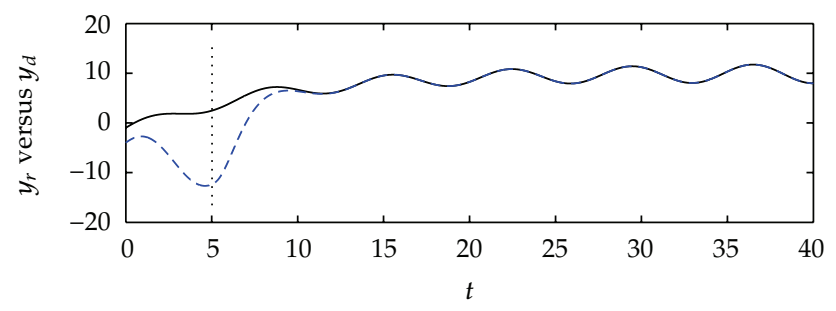

$-y_{d}$

(b)

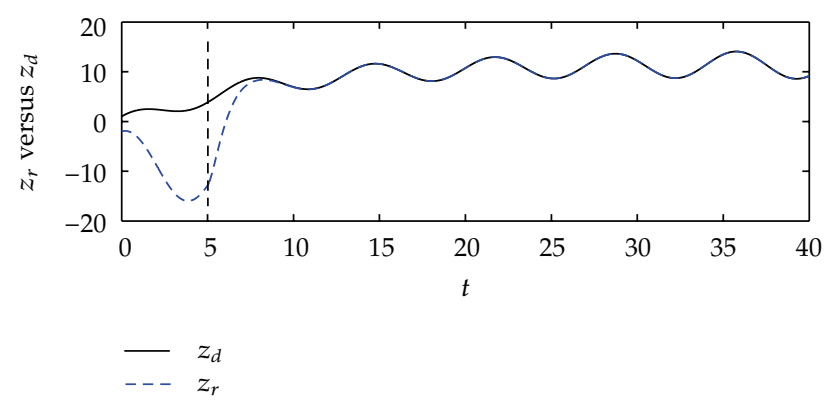

(c)

Figure 8: Plots of the perturbed B-VDP oscillators' state trajectories versus time when the sliding mode control is used.

ensuring complete synchronization between the two systems. Furthermore, a nonlinear transformation is used to transform the error dynamics into the controller canonical form; this transformation simplifies the design of the sliding mode controller. Simulation studies show the effectiveness of the proposed control schemes.

\section{References}

[1] P. Glendinning, Stability, Instability and Chaos: An Introduction to the Theory of Nonlinear Differential Equations, Cambridge University, Cambridge, UK, 1994.

[2] Q. A. Memon, "Synchronized choas for network security," Computer Communications, vol. 26, no. 6, pp. 498-505, 2003. 
[3] R. E. Mirollo and S. Strogatz, "Synchronization of pulse-coupled biological oscillators," SIAM Journal on Applied Mathematics, vol. 50, no. 6, pp. 1645-1662, 1990.

[4] H. Nijmeijer and H. Berghuis, "On Lyapunov control of the Duffing equation," IEEE Transactions on Circuits and Systems, vol. 42, no. 8, pp. 473-477, 1995.

[5] S. S. Ge, C. Wang, and T. H. Lee, "Adaptive backstepping control of a class of chaotic systems," International Journal of Bifurcation and Chaos in Applied Sciences and Engineering, vol. 10, no. 5, pp. 1149-1156, 2000.

[6] F. M. Kakmeni, S. Bowong, C. Tchawoua, and E. Kaptouom, "Chaos control and synchronization of a $\Phi^{6}$-van der pol oscillator," Physics Letters A, vol. 322, no. 5-6, pp. 305-323, 2004.

[7] H. Fotsin and S. Bowong, "Adaptive control and synchronization of chaotic systems consisting of van der pol oscillators coupled to linear oscillators," Chaos, Solitons and Fractals, vol. 27, no. 3, pp. 822-835, 2006.

[8] L. Zhao, L. Xue, and J. Wang, "Variable structure control for synchronization of chaotic systems with structure or parameters mismatching," in Proceedings of the 6th World Congress on Intelligent Control and Automation (WCICA '06), vol. 1, pp. 2106-2110, Dalian, China, 2006.

[9] U. E. Vincent, "Chaos synchronization using active control and backstepping control: a comparative analysis," Nonlinear Analysis, vol. 13, no. 2, pp. 253-261, 2008.

[10] S. Dadras and H. R. Momeni, "Adaptive sliding mode control of chaotic dynamical systems with application to synchronization," Mathematics and Computers in Simulation, vol. 80, no. 12, pp. 22452257, 2010.

[11] X. Jian, "Anti-synchronization of Lienard chaotic systems via feedback control," International Journal of Physical Sciences, vol. 5, no. 18, pp. 2757-2761, 2010.

[12] A. N. Njah and U. E. Vincent, "Synchronization and anti-synchronization of chaos in an extended Bonhoffer-van der pol oscillator using active control," Journal of Sound and Vibration, vol. 319, no. 1-2, pp. 41-49, 2009.

[13] M. Chen, C. Jiang, B. Jiang, and Q. Wu, “Sliding mode synchronization controller design with neural network for uncertain chaotic systems," Chaos, Solitons and Fractals, vol. 39, no. 4, pp. 1856-1863, 2009.

[14] E. M. Elabbasy and M. M. El-Dessoky, "Synchronization of van der pol oscillator and Chen chaotic dynamical system," Chaos, Solitons and Fractals, vol. 36, no. 5, pp. 1425-1435, 2008.

[15] K. D. Young, V. I. Utkin, and U. Ozguner, "A control engineer's guide to sliding mode control," IEEE Transactions on Control Systems Technology, vol. 7, no. 3, pp. 328-342, 1999.

[16] W. Perruquetti and J. P. Barbot, Sliding Mode Control In Engineering, Marcel Dekker, 2002.

[17] J. E. Slotine and W. Li, Applied Nonlinear Control, Prentice-Hall, 1991. 


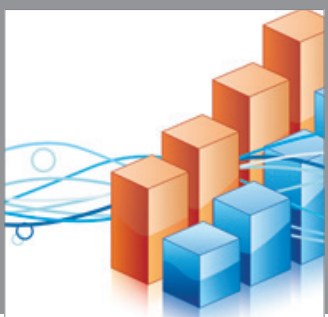

Advances in

Operations Research

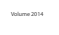

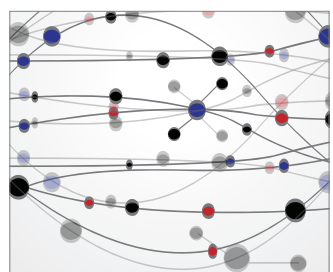

\section{The Scientific} World Journal
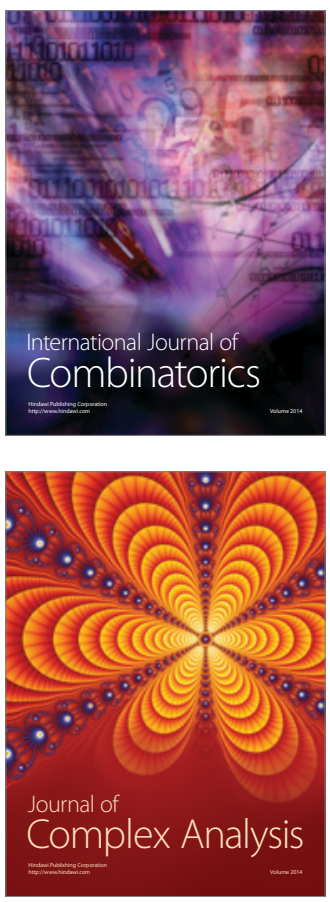

International Journal of

Mathematics and

Mathematical

Sciences
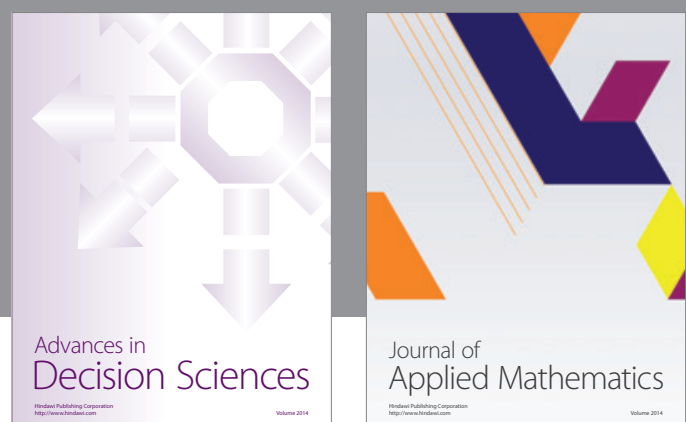

Journal of

Applied Mathematics
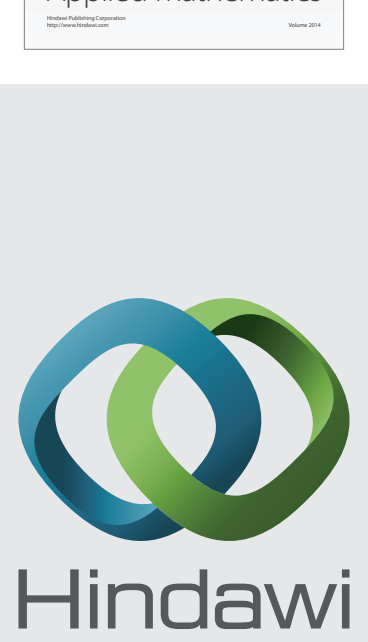

Submit your manuscripts at http://www.hindawi.com
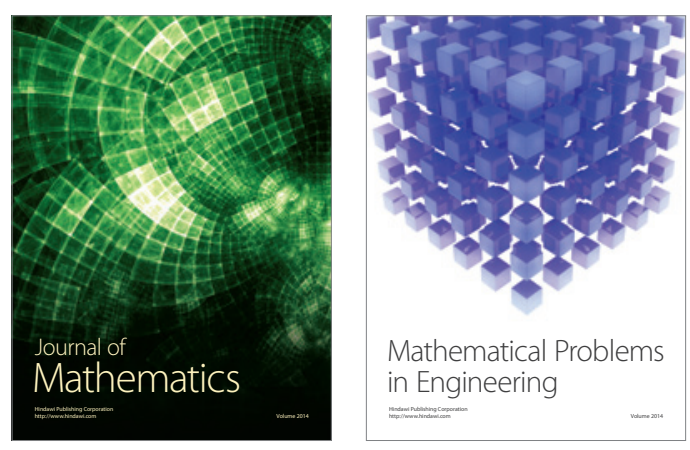

Mathematical Problems in Engineering
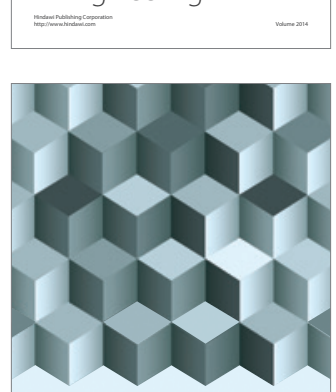

Journal of

Function Spaces
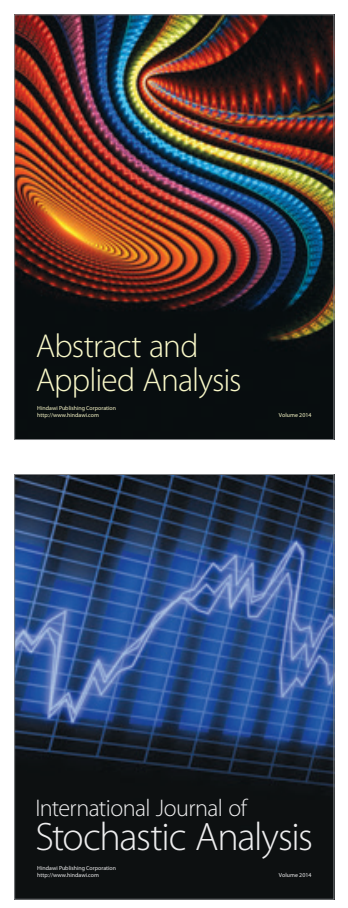

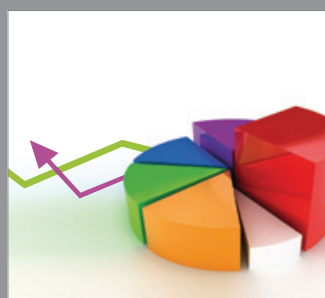

ournal of

Probability and Statistics

Promensencen
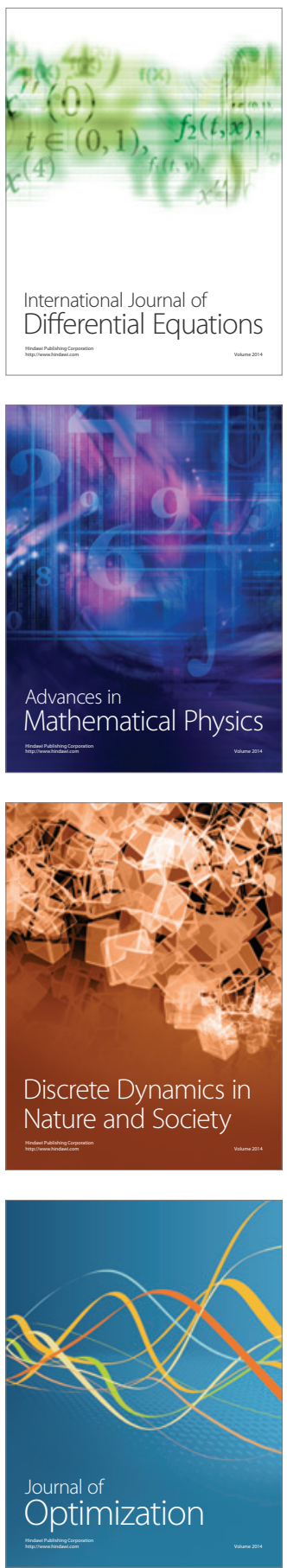